\title{
Eulerian-Lagrangian simulations of settling and agitated dense solid-liquid suspensions - achieving grid convergence
}

\author{
J.J. Derksen \\ School of Engineering, University of Aberdeen, Aberdeen, UK \\ jderksen@abdn.ac.uk
}

Submitted to AIChE Journal - July 2017

Revision submitted: October 2017 - Changes in blue font

\section{Abstract}

Eulerian-Lagrangian simulations of solid-liquid flow have been performed. The volume-averaged NavierStokes equations have been solved by a variant of the lattice-Boltzmann method; the solids dynamics by integrating Newton's second law for each individual particle. Solids and liquid are coupled via mapping functions. The application is solids suspension in a mixing tank operating in the transitional regime (the impeller-based Reynolds number is 4,000), an overall solids volume fraction of $10 \%$ and a particle-liquid combination with an Archimedes number of 30. In this application, the required grid resolution is dictated by the liquid flow and we thus need freedom to choose the particle size independent of the grid spacing. Preliminary hindered settling simulations show that the proposed Eulerian-Lagrangian mapping strategy indeed offers this independence. The subsequent mixing tank simulations generate grid-independent results.

\section{Keywords}

Solid-liquid suspension, lattice-Boltzmann method, discrete particle method, hindered settling, two-way coupling, agitated suspensions 


\section{Introduction}

Mixing tanks with the purpose of suspending solid particles in a liquid are a common feature in chemical and biochemical industrial processes. The applications are wide-ranging: from wastewater treatment to food processing; from catalytic slurry reactors to industrial crystallization devices. Solid-liquid mass transfer - in many cases including surface reactions - is an important objective of the process steps carried out in the mixing equipment. Since mass transfer strongly depends on the extent to which the surface of the solid particles is exposed to liquid flow, the fluid and solids dynamics are directly relevant for process performance. Also for characterizing natural processes such as sediment transport in rivers and coastal areas, the dynamics of solid particles in liquid flow is a feature demanding accurate description and thorough understanding. These notions have led to extensive research on the dynamic behavior of solid-liquid suspensions.

Next to theoretical and experimental approaches dating back to the seminal works of Stokes [1], Richardson \& Zaki [2] and - in the field of mixing tanks - Zwietering [3] , computational methods are a means of researching the dynamics of suspensions. There is no universal numerical method to simulate suspension flow. The approach depends on the questions asked, and the computational resources available. An important division is the one between an Eulerian-Eulerian (EE) and an EulerianLagrangian (EL) viewpoint. In an EE simulation, the solids phase is described as a continuum, governed by continuum forms of mass and momentum balance equations. In an EL simulation, particles are tracked individually or as clusters (parcels) through the liquid based on Newton's second law and hydrodynamic and other forces.

This paper will exclusively consider the EL approach. We focus on EL simulations since in subsequent research we want to quantify mass transfer at the particle level, i.e. individual particles will be followed on their way through the liquid, thereby keeping track of the extent to which they exchange mass with their surroundings. If mass transfer would involve change in particle size, EL simulations then also would naturally allow simulating the evolution of a particle size distribution in the course of a 
process, something which is much harder to do in an EE context. For the remainder of this paper, however, mass transfer will not be considered. The main flow system that will be considered in this paper is a mixing tank, operating in the mildly turbulent / transitional regime (to be specified below in a quantitative sense) such that the liquid flow can be simulated directly, without the need for a turbulence closure model or subgrid scale model.

Within the realm of EL approaches, a distinction needs to be made between particle-resolved, and particle-unresolved simulations. In particle-resolved simulations, the resolution of the Eulerian grid on which the fluid flow is solved is sufficiently high to explicitly apply the no-slip condition at the surface of the particles and thus in detail calculate the flow around them individually [4-8]. This way, hydrodynamic forces and torques on the particles are directly determined and used to solve the translational and rotational equations of motion of the particles. This level of detail requires fine grids and thus extensive, usually parallel, computational resources and efficient codes. Currently simulations with up to 1 million resolved particles have been reported [8]. Even in lab-scale flow systems, however, this number of particles is easily exceeded. For dealing with such systems, one then needs to revert to methods that are less resolved at the particle level: particle-unresolved simulations.

Particle-unresolved simulations come with a number of issues that are the subject of active research.

(1) Determination of hydrodynamic forces and torques on the particles. Since the flow around the particles is not resolved, one needs closure relations for hydrodynamic forces and torques on the particles as a function of local conditions, usually expressed in terms of a Reynolds number based on the slip velocity between particle and surrounding fluid, and the local solids volume fraction $[9,10]$. Additional (dimensionless) parameters that have been considered in force expressions are the Stokes number for dealing with inertia and with the suspension's micro structure [11], and a Reynolds number based on granular temperature for dealing with the effects of fluctuations [12]. One emphasis of current research is on closure relations for the drag force. In gas-solid systems, the drag force is the dominant hydrodynamic 
force [13]. In liquid-solid systems, however, additional effects such as lift, added mass, and history effects [14] might be relevant as well.

(2) The exchange of information between grid-based (Eulerian) quantities and particle-based (Lagrangian) quantities. Examples are the determination of the Eulerian solids volume fraction field $\phi$ (relevant for solving the volume-averaged fluid equations, see Eqs. 1 and 2 below) from the (off-grid) locations of individual particles, as well as the fluid velocity in the direct vicinity of a particle from the velocity distribution on the grid. This Eulerian-Lagrangian exchange is facilitated by mapping functions that distribute Lagrangian quantities over the grid, and generate weighted averages of Eulerian quantities at the center location of a particle [15].

The modestly turbulent mixing tank applications we are interested in have specific requirements for the mapping process: It should be able to deal with particle sizes $(d)$ that are of the same order of magnitude as the grid spacing $\Delta ; d=O(\Delta)$. Where some, largely interpolation based, mapping methods require the mesh to be much wider than the particle size [16], there is recent development in mappings facilitating $d=O(\Delta)$ simulations [17,18]. We need such mappings to have freedom in the choice of grid spacing to resolve the transitional or turbulent flow in the mixing tank. Ideally the choice of grid spacing is independent of the particle size and mainly determined by requirements for sufficiently resolving the liquid flow. The aim of this work is to establish grid-independent simulations of solid-liquid flow that are high on solids loading (overall solids volume fraction of order 10\%) with an unresolved - mapping-based - particle approach. We use the same mapping procedure that was tested in a previous paper for fully periodic, three-dimensional systems [18]. This latter study allowed to compare average slip velocities and velocity fluctuation levels (of liquid and solids) obtained with particle-unresolved procedures to fully resolved simulations of the same systems and thus benchmark / optimize the unresolved procedure.

First in this paper, we apply the simulation procedure to the case of particles settling in liquid in a column towards a solid bottom. This mimics the classical Richardson \& Zaki experiments [2], and enables performing a number of basic checks (hindered settling speeds, build-up of a hydrostatic pressure 
gradient, velocity fluctuation levels, grid effects) on the simulation procedure. Then we simulate - at various resolution levels - the flow in a mixing tank with zero-velocity initial conditions and the particles forming a granular bed on the tank bottom. After starting the impeller we keep track of the suspension process and continue beyond the time frame over which quasi steady state is reached. The simulation conditions are chosen such that they are amenable to lab-scale visualization experiments with refractive index matching of solids and liquid [19]. The impeller-based Reynolds number is 4,000, the Archimedes number associated to particles and liquid is $\mathrm{Ar} \equiv \frac{g \Delta \rho d^{3}}{\rho v^{2}} \approx 30$ (with $g$ gravitational acceleration, $\Delta \rho=\rho_{s}-\rho$ the difference between solid and liquid density, and $v$ the kinematic viscosity of the liquid), and the solid-over-liquid density ratio $\rho_{s} / \rho$ is in the range $2.23-2.5$.

In the subsequent sections of this paper we first introduce the flow systems. We then summarize the simulation procedure and refer to the literature (e.g. $[18,20])$ for further details. In discussing the hindered settling results we focus on the impact of model choices on the settling speed. A study of grid effects is the main theme when mixing tank simulations are presented. In the final section we draw conclusions and give an outlook to further study.

\section{Flow systems and simulation methods}

\subsection{Flow systems}

The flow domains are rectangular, three-dimensional volumes of size $n x \times n y \times n z$. Gravity points in the negative $z$-direction: $\mathbf{g}=-g \mathbf{e}_{\mathbf{z}}$. The domain size in the horizontal directions are the same: $n x=n y$. The systems in which we study hindered settling have periodic conditions in the $x$ and $y$ directions and solid planar walls at the top and bottom. The agitated tanks are rectangular as well and have solid walls all around. Agitation is achieved by spinning an impeller with four blades, pitched under $45^{\circ}$ in a direction such that fluid is pumped downward. Figure 1 provides the geometrical details of the mixing tank. The Reynolds number associated to the flow induced by the impeller is defined as $\operatorname{Re}_{m x} \equiv N D^{2} / v$ with $D$ the 
impeller diameter and $N$ the impeller speed (in revolutions per unit time). The flow systems contain a Newtonian liquid with density $\rho$ and kinematic viscosity $v$ and spherical solid particles of equal size with diameter $d$ and density $\rho_{s}$ larger than $\rho$.

There are various ways to define the flow conditions in the systems as introduced above. The dimensionless numbers we use to characterize the hindered settling systems are the average solids volume fraction $\langle\phi\rangle$ in the part of the volume loaded with particles, the density ratio $\rho_{s} / \rho$, and the singleparticle settling Reynolds number $\operatorname{Re}_{\infty} \equiv u_{\infty} d / v$ with $u_{\infty}$ the settling velocity that we determine from a force balance over a single particle in an infinite domain $g\left(\rho_{s}-\rho\right) \pi d^{3} / 6=\frac{1}{2} C_{D} \rho u_{\infty}^{2} \pi d^{2} / 4$. For the drag coefficient $C_{D}$ the Schiller-Naumann correlation [21] $C_{D}=24\left(1+0.15 \mathrm{Re}^{0.687}\right) / \mathrm{Re}$ is applied. For the solid-liquid mixing simulations, next to the impeller-based Reynolds number $\operatorname{Re}_{m x}$ and the density ratio $\rho_{s} / \rho$ there is the Shields parameter $\theta=\frac{\rho N^{2} D^{2}}{g\left(\rho_{s}-\rho\right) d}$. The latter expresses the competition between inertial stress generated by the impeller motion suspending the particles (that scales with $\rho N^{2} D^{2}$ ), and net gravity pulling them down [6].

\subsection{Liquid and solids dynamics}

Fluid flow is solved on a three-dimensional Eulerian grid. The Eulerian grid is uniform and cubic with grid spacing $\Delta$. The spherical particles that move through this grid have a diameter comparable to $\Delta$; the range of diameters investigated in this paper is $0.77 \leq d / \Delta \leq 3.3$. On the Eulerian grid the volumeaveraged continuity equation and momentum balance for the liquid phase [22,23] are solved:

$$
\begin{gathered}
\frac{\partial}{\partial t}\left(\rho \phi^{c}\right)+\nabla \cdot\left(\rho \phi^{c} \mathbf{u}\right)=0 \\
\frac{\partial}{\partial t}\left(\rho \phi^{c} \mathbf{u}\right)+\nabla \cdot\left(\rho \phi^{c} \mathbf{u u}\right)=\phi^{c} \nabla \cdot \boldsymbol{\pi}+\mathbf{f}_{\mathrm{s}}
\end{gathered}
$$


with $\phi^{c} \equiv 1-\phi$ the continuous phase (liquid) volume fraction and $\phi$ the solids volume fraction, $\mathbf{u}$ the interstitial liquid velocity, $\boldsymbol{\pi}$ the liquid's stress tensor, and $\mathbf{f}_{\mathrm{s}}$ the force per unit volume the solid particles exert on the liquid. Equations 1 and 2 are solved with a variant of the lattice-Boltzmann method. Full details can be found in $[18,20]$.

The dynamics of the spherical solid particles is governed by Newton's equations of motion

$$
\begin{gathered}
\rho_{s} \frac{\pi}{6} d^{3} \frac{d \mathbf{u}_{\mathbf{p}}}{d t}=\mathbf{F}_{\mathbf{h}}+\mathbf{F}_{\mathbf{c}}-\frac{\pi}{6} d^{3}\left(\rho_{s}-\rho\right) g \mathbf{e}_{\mathbf{x}} \\
\rho_{s} \frac{\pi}{60} d^{5} \frac{d \boldsymbol{\omega}_{\mathbf{p}}}{d t}=\mathbf{T}_{\mathbf{h}}+\mathbf{T}_{\mathbf{c}}
\end{gathered}
$$

and by

$$
\frac{d \mathbf{x}_{\mathbf{p}}}{d t}=\mathbf{u}_{\mathbf{p}}
$$

with $\mathbf{u}_{\mathbf{p}}, \boldsymbol{\omega}_{\mathbf{p}}, \mathbf{x}_{\mathbf{p}}$ the linear velocity, angular velocity, and center location of a spherical particle respectively (note that - because we are dealing with spheres - there is no need to track the angular "location" of the particles), $\mathbf{F}_{h}$ and $\mathbf{T}_{\mathbf{h}}$ the hydrodynamic force and torque on a particle, and $\mathbf{F}_{\mathbf{c}}$ and $\mathbf{T}_{\mathbf{c}}$ the contact force and torque due to particle-particle collisions and lubrication effects.

\subsection{Modelling assumptions and implementation}

The only hydrodynamic force on the particles we will be considering is the drag force. For liquid-solid systems - with density ratios of order one - additional hydrodynamic effects such as lift, added mass, and history forces might have a significant effect [14]. At this stage we discard these effects. Eventually, experimental data and sensitivity analyses through simulations will need to shed light on the importance of additional forces under specific flow conditions.

The drag force is written in the form

$$
\mathbf{F}_{\mathbf{D}}=3 \pi \rho v d\left(\mathbf{u}-\mathbf{u}_{\mathbf{p}}\right) F(\operatorname{Re}, \phi)
$$


with $\operatorname{Re}=(1-\phi)\left|\mathbf{u}-\mathbf{u}_{\mathbf{p}}\right| d / v$.

An additional simplification thus is that drag only depends on the solids volume fraction, and on the Reynolds number. That is, we do not include terms in the drag expression that depend on the granular temperature (as in [12]), or on the Stokes number [11]. The function $F$ is written as a product function $F(\operatorname{Re}, \phi)=p(\operatorname{Re}) q(\phi)$. The Reynolds dependency is captured through the Schiller-Naumann correlation [21] $p(\operatorname{Re})=\left(1+0.15 \operatorname{Re}^{0.687}\right)$ (which is valid for $\operatorname{Re}<1,000$, a condition met in this paper). For the dependence of drag on the local solids volume fraction we have tested two expressions: the Wen $\& \mathrm{Yu}$ expression $q(\phi)=(1-\phi)^{-\beta}$ with $\beta=2.65$ [24] and the Van der Hoef et al expression $q(\phi)=\frac{10 \phi}{(1-\phi)}+(1-\phi)^{3}\left(1+\frac{3}{2} \sqrt{\phi}\right)[25]$. As has been noticed [11], the latter expression results in higher values for the drag force as compared to the former. In [11] this has been identified as an effect of the Stokes number. The Wen \& Yu correlation has been derived from hindered settling experiments in solidliquid systems that have moderate Stokes numbers. The Van der Hoef et al expression is the result of simulations of the flow around static, random assemblies of particles. This is a system characteristic for infinite Stokes numbers. Since the solids are static and thus would take "infinite time" to change configuration, the fluid phase time scales are infinitely smaller than those of the solids.

The force exerted by the fluid on the particle is the sum of $\mathbf{F}_{\mathbf{D}}$ and the contribution from a slowly varying stress field (e.g. due to buoyancy) around the particle. This total hydrodynamic force on the particle as it shows up in Eq. 3 can be expressed as $\mathbf{F}_{\mathbf{h}}=\mathbf{F}_{\mathbf{D}} /(1-\phi)$ [26]. One manifestation of a varying stress field around the particles is the pressure that builds up as a consequence of the net weight of the collection of particles. As will be shown, this results in a pressure gradient $\frac{\partial p}{\partial z}=-g\left(\rho_{m}-\rho\right)$ with $\rho_{m}=\phi \rho_{s}+(1-\phi) \rho$ the mixture density. In this expression for the vertical pressure gradient, the liquid density $\rho$ is subtracted since Eq. 3 already accounts for the liquid-only buoyancy force. 
The body force $\mathbf{f}_{\mathrm{s}}$ in Eq. 1 is the reaction of the drag force on the fluid. Feeding back the drag force on the fluid is an example of mapping: relating Lagrangian properties (in this case drag force $\mathbf{F}_{\mathbf{D}}$ ) to Eulerian properties $\left(\mathbf{f}_{\mathrm{s}}\right)$.

The one-dimensional version of the mapping function used in this work reads

$$
\begin{aligned}
& \mu(\xi)=\frac{15}{16}\left[\frac{\xi^{4}}{\lambda^{5}}-2 \frac{\xi^{2}}{\lambda^{3}}+\frac{1}{\lambda}\right] \text { for }-\lambda \leq \xi \leq \lambda \\
& \mu(\xi)=0 \text { for }|\xi|>\lambda
\end{aligned}
$$

This is a "clipped fourth-order polynomial" [27] with $\lambda$ the half-width of the mapping function. It shows resemblance to a Gaussian distribution but is computationally more efficient to calculate than a Gaussian and is zero at $\pm \lambda$. To determine some property $\alpha$, that is known on the Eulerian grid, at a Lagrangian location $\kappa$, the product of mapping function and property is integrated: $\langle\alpha(\kappa)\rangle_{\lambda}=\int_{-\lambda}^{\lambda} \mu(\xi-\kappa) \alpha(\xi) d \xi$. The property $\alpha(\xi)$ is defined on the equidistant Eulerian grid $\xi_{i}$ with spacing $\Delta$ by values $\alpha_{i}$. We approximate $\alpha(\xi)$ in the integrant as a stair-step function, i.e. $\alpha(\xi)=\alpha_{i}$ for $\xi_{i}-\frac{1}{2} \Delta \leq \xi<\xi_{i}+\frac{1}{2} \Delta$. Given the discrete nature of $\alpha(\xi)$, the integral can be written as $\langle\alpha(\kappa)\rangle_{\lambda}=\sum_{i} \eta_{i} \alpha_{i}$ with $\eta_{i}$ coefficients following from integrating the mapping function. The extension to a three-dimensional Eulerian grid and a three dimensional Lagrangian location $\boldsymbol{\kappa}$ is straightforward and can be written as $\langle\alpha(\mathbf{\kappa})\rangle_{\lambda}=\sum_{i} \sum_{j} \sum_{k} \eta_{i j k} \alpha_{i j k}$ with $i, j, k$ discrete coordinates in $x, y$, and $z$-direction respectively. The coefficients $\eta_{i j k}$ are only non-zero on grid points within a volume of $(2 \lambda)^{3}$ around $\kappa$. Also $\sum_{i} \sum_{j} \sum_{k} \eta_{i j k}=1$ since in case $\alpha$ is uniform, $\langle\alpha\rangle_{\lambda}=\alpha$. For efficient calculations, we use a look-up table for determining the coefficients $\eta_{i j k}$. Prior to a simulation all coefficients $\eta_{i j k}$ are determined for a three-dimensional grid of Lagrangian points $(10 \times 10 \times 10$ points in our code $)$ in a grid cell. During the actual simulation, the coefficients associated to a specific Lagrangian location (a particle) are obtained from tri-linear 
interpolation in this grid of points. Interpolation guarantees smooth time-variation of the mapping operations.

The coefficients $\eta_{i j k}$ are used to distribute Lagrangian properties to the grid. As an example, the drag force on one of the particles ( $\mathbf{F}_{\mathbf{D}}$ ) contributes to the body force on the fluid $\mathbf{f}_{\mathrm{s}}$ (see Eq. 2) in grid cell $i, j, k$ by an amount $-\frac{1}{\Delta^{3}} \mathbf{F}_{\mathbf{D}} \eta_{i j k}$.

At three instances in the simulation procedure mapping operations are applied: (1) to determine the liquid velocity $\mathbf{u}$ (to be used in Eq. 6 to determine the drag force) at the location of the particle from the Eulerian velocity field; (2) to determine the Eulerian solids volume fraction field $\phi$ from the location and size of the particles so that $\phi^{c}=1-\phi$ is a known field when solving Eqs. 1 and 2; (3) (as explained above) to determine the Eulerian vector field $\mathbf{f}_{\mathrm{s}}$ from the drag forces $\mathbf{F}_{\mathbf{D}}$ on the particles.

The choice of the width of the mapping function $(\lambda)$ is worthwhile investigating. Earlier research $[17,18]$ suggests a value of $\lambda=1.5 d$ and we will be using this as our default choice. However, we will be looking into the effects of excursions from this choice.

\subsection{Particle dynamics}

Equations 3-5 describe the dynamics of the particles. The way $\mathbf{F}_{\mathbf{h}}$ (in Eq. 3) has been determined was shown above. The contact force $\mathbf{F}_{\mathbf{c}}$ consists of two parts: soft-sphere collision forces $\mathbf{F}_{\mathrm{ssc}}$ and lubrication forces $\mathbf{F}_{\text {lub }}$. Both forces are assumed to be radial forces. This means that they act on the line connecting the two sphere centers involved in a contact. The collisions thus are assumed to be smooth so that we will not be considering tangential contact forces and contact torques, as a result $\mathbf{T}_{\mathbf{c}}=\mathbf{0}$ in Eq. 4 .

The soft-sphere collision force is a radial repulsive force proportional to the distance $\delta$ over which the spheres overlap: $\left|\mathbf{F}_{\text {ssc }}\right|=\left(\pi^{2} m / t_{c}^{2}\right) \delta$ with $m=\pi \rho_{s} d^{3} / 6$ the mass of a particle, and $t_{c}$ a parameter that controls the typical time of contact between two particles [11]. Particle-wall collisions are treated similar 
to particle-particle collisions: a fixed, fictitious particle is placed at the opposite side of the wall and the actual particle bounces smoothly with the fictitious particle.

Lubrication forces occur when two closely spaced particles move relative to one another. The radial component of the lubrication force (the only component considered here) is the result of a draining liquid film between two approaching particles, and a liquid film filling upon separation. For low Reynolds number film flow, the radial lubrication force on particle $j$ due to particle $i$ can be written as $\mathbf{F}_{\text {lub,j }}=F_{l u b} \mathbf{n}_{\mathbf{i j}}$ with $\mathbf{n}_{\mathbf{i j}}=\left(\mathbf{x}_{\mathbf{p}, \mathbf{j}}-\mathbf{x}_{\mathbf{p}, \mathbf{i}}\right) / \mathbf{x}_{\mathbf{p}, \mathbf{j}}-\mathbf{x}_{\mathbf{p}, \mathbf{i}} \mid$ the unit vector along the line connecting the centers of the two particles, and $F_{l u b}=-\frac{3}{8} \pi v \rho d^{2}\left(\mathbf{u}_{\mathbf{p}, \mathbf{j}}-\mathbf{u}_{\mathbf{p}, \mathbf{i}}\right) \cdot \mathbf{n}_{\mathbf{i j}} / s$ with $s$ the minimum distance between particle surfaces [28]. The force on particle $i$ due to $j$ is opposite: $\mathbf{F}_{\text {lub, } \mathbf{i}}=-F_{l u b} \mathbf{n}_{\mathbf{i j}}$. In the simulations these expressions have been modified in two ways. (1) A cut-off distance of $0.1 d$ has been introduced: for $s \geq 0.1 d$ the lubrication force is zero, for $s<0.1 d F_{l u b} \propto 1 / s-10 / d$ [26]. (2) The lubrication force saturates if $s \leq 10^{-3} d$ [26].

Since collisions between particles and between particles and walls are smooth, the only source of rotation is the hydrodynamic torque $\mathbf{T}_{\mathbf{h}}$ (in Eq. 4). It is determined according to a creeping flow approximation: $\mathbf{T}_{\mathbf{h}}=\pi \rho v d^{3}\left(\frac{1}{2} \boldsymbol{\omega}-\boldsymbol{\omega}_{\mathbf{p}}\right)$ with $\boldsymbol{\omega}$ the vorticity of the liquid in the direct vicinity of the particle [29]. The hydrodynamic torque is not fed back to the liquid. As a result, the rotation of the particles has no impact on the overall dynamics of the two-phase flow.

The equations of motion (Eq. 3-5) are solved by means of a split derivative time integration which has been discussed in detail in [30]. Such integration enhances stability which is useful in case of modest solid over liquid density ratios, as we have in this paper.

As a summary, we here list the main choices, assumptions and limitations of the proposed simulation procedure: (a) Drag is the only hydrodynamic force; it depends on a particle-based Reynolds number and local solids volume fraction. (b) Mapping functions with half-width $\lambda=1.5 d$ are used to relate Eulerian and Lagrangian flow properties; we will investigate the sensitivity with respect to $\lambda / d$. (c) Collisions are smooth, and interaction forces (soft-sphere and lubrication) are radial. (d) The torque on a 
particle is estimated based on a creeping flow assumption and particle rotation is not fed back to the liquid flow.

\section{Results}

\subsection{Hindered settling}

The hindered settling base-case consists of a liquid filled domain, periodic in the horizontal directions $x$ and $y$ and enclosed between a top and bottom solid wall; $n x=n y=54.5 d ; n z=109 d$. A total of 62,500 solid particles of diameter $d$ are initialized randomly in the lower part of the domain, in between $z=0.5 d$ and $z=0.4 n z$ such that in this region $\langle\phi\rangle=0.252$. The density ratio is $\rho_{s} / \rho=2.50$. Viscosity and gravitational acceleration are such that $\mathrm{Re}_{\infty}=2.89$. The default settings apply for the half-width of the mapping function $(\lambda / d=1.5)$, the hindered settling function $\left(q(\phi)=(1-\phi)^{-2.65}\right)$ and the collision time $\left(t_{c} v / d^{2}=0.0083\right)$

At moment zero the particles are released and start settling. In Figure 2 we show snapshots of vertical cross sections through the domain in terms of liquid and solids velocity vectors for two resolutions: $d=1.1 \Delta$ and $2.2 \Delta$. The liquid develops vortical structures as it gets agitated by the settling solids. Since we start the simulations at the two resolutions with the same initial solids configuration, there is strong similarity between the panels in Figure 2. On average one observes an upward liquid motion to compensate for downward particle volume flux. The interface between particle-laden and clear liquid is sharp, also after - as in Figure 2 - significant time has elapsed since the start of the settling process.

The system develops a vertical pressure gradient due to the net weight $g \pi\left(\rho_{s}-\rho\right) d^{3} / 6$ of the particles with $\frac{\partial p}{\partial z}=-g\left(\rho_{m}-\rho\right)$ where $\rho_{m}=\phi \rho_{s}+(1-\phi) \rho$ is the mixture density. We thus expect a pressure gradient $\frac{\partial p}{\partial z}=-\phi g\left(\rho_{s}-\rho\right)$; in the scaling of Figure 3 this is $\frac{d}{g\left(\rho_{s}-\rho\right) d} \frac{\partial p}{\partial z}=-\phi$. This is what 
is observed in the left panel of Figure 3: the slope of the pressure profile in the part of the volume that contains settling particles is approximately -0.25 which is minus the average solids volume fraction $\langle\phi\rangle$ there. The solids volume fraction contours in Figure 3 are consistent with the pressure profile: approximately zero pressure gradient in the clear liquid and in the settled granular bed on the bottom wall, and a constant, negative gradient in the region where the particles settle.

Hindered settling speeds have been determined as it would have been done in an experiment: measure the vertical location of the interface between suspension and clear liquid as a function of time. As we observed in Figures 2 and 3, the interface is well-defined and horizontal. Two different ways of quantifying the interface location have been tested: (1) the laterally ( $x$ and $y$ ) averaged $z$ location where the Eulerian solids volume fraction field is half the solids volume fraction in the suspension; (2) the average $z$ location of the top $2 \%$ of the particles. In Figure 4 it shows that the results of two methods have close resemblance. The settling speeds as presented in the remainder of this section have been determined with the second (top $2 \%$ ) method by fitting a straight line to the linear portion of the time series as this method shows a slightly smoother time series (see Figure 4).

In Figure 5 it is demonstrated that the simulation procedure mimics the dependency of the settling velocity on the average solids volume fraction as proposed by Richardson \& Zaki [2] quite well (left panel of Figure 5). In a more critical test we compare hindered settling in terms of the exponent $n$ in the settling function $\left(u_{s} / u_{\infty}=(1-\langle\phi\rangle)^{n}\right)$ and the way it depends on a Reynolds number $u_{s} d / v$ with an empirical correlation due to Di Felice [31]. The simulations show an $n$ that is some $10 \%$ lower than the empirical correlation. The weakly downward trend in $n$ with respect to the Reynolds number is represented correctly by the simulations.

In our previous work [18] it was shown that average slip velocities were virtually insensitive for the half-width of the mapping function $\lambda$ as long as $\lambda / d \geq 1.5$. The left panel of Figure 6 confirms this for the current hindered settling simulations. More importantly, however, the spatial resolution of the simulations expressed as $d / \Delta$ at fixed $\lambda / d=1.5$ has virtually no effect on the settling velocity (see the 
right panel of Figure 6). It implies that - at least for average settling speeds - there is freedom in choosing spatial resolution relative to the particle size, at least in the range $1 \leq d / \Delta \leq 3$. The situation for fluctuating velocities is more complicated in the case of the present simulations. Where the settling speed is steady in a significant part of the time window of a simulation (see Figure 4), the per-particle variability in the velocity (expressed in a root-mean-square value) is a transient as shown in Figure 7 . The rootmean-square (rms) values are - as expected - larger for the vertical velocity component than for the horizontal components (by approximately a factor of 2) [20,32]. The dependency of the rms particle velocity values with respect to the width of the mapping function follow the same trend as in the (fully periodic) simulations in [18]: the wider the mapping function, the weaker the rms velocity values (in [18] it was argued that for $\lambda / d \rightarrow \infty$ fluctuations would disappear). From comparison with particle-resolved simulations, $\lambda / d=1.5$ was found to be the mapping function width that best mimicked the particle resolved simulations [18], in line with conclusions drawn in [17]. With the latter value for $\lambda / d$ the sensitivity of the rms velocities with respect to $d / \Delta$ was assessed, see the right panel of Figure 7 . The outlier in this panel is the simulation with the lowest resolution (at $d / \Delta=0.77$ ). As long as $d / \Delta \geq 1.1$, the resolution of the particles on the grid has no significant impact on the rms particles velocity values.

In summary, the mapping procedure, in combination with the lattice-Boltzmann based numerical scheme, shows for hindered settling towards a solid wall results that are largely independent on the level of resolution of the particles on the grid, as expressed through the ratio of particle size and grid spacing $d / \Delta$. It is important to realize that the most appropriate choice of $\lambda / d=1.5$ is based on a limited range of solids volume fractions and (particle-based) Reynolds numbers. It might very well be - and some of the comparisons with particle resolved simulations as presented in [18] indeed suggest so - that the choice of $\lambda / d=1.5$ is regime dependent. In the subsequent section, the numerical procedure will be applied to a mixing tank configuration where, next to determining particle dynamics, also resolving the complex flow generated by the impeller imposes demands on the grid spacing. 


\subsection{Agitated solid-liquid flow}

The dimensionless numbers we use for defining the agitated flow in the mixing tank (with geometry and aspect ratios as given in Figure 1) have values $\operatorname{Re}_{m x} \equiv N D^{2} / v=4,000, \quad \theta=\frac{\rho N^{2} D^{2}}{g\left(\rho_{s}-\rho\right) d}=260$, and $\rho_{s} / \rho=2.23$. The particle size relative to the impeller diameter is $d / D=0.0208$; the tank-averaged solids volume fraction, i.e. total volume of solids over total tank volume is $\langle\phi\rangle=0.098$; the number of particles is 250,000 . A Stokes number with $1 /(4 N)$ (the inverse of the impeller blade passage frequency) is defined as $\mathrm{St} \equiv \frac{2}{9} \frac{\rho_{s}}{\rho} \frac{d^{2} 4 N}{v}$ and has value of 3.4, i.e. an intermediate Stokes number.

The main purpose of this study of agitated solid-liquid flow is to establish grid independence. Sufficiently fine grids are required to resolve the flow at the given - impeller-based - Reynolds number. The settling simulations have shown that, with the proposed mapping procedure, there is freedom in the choice of the particle diameter relative to the grid spacing. In the right panels of Figure 6 and 7 it is shown that results on respectively settling speed and particle velocity fluctuations during settling are not sensitive to the particle size relative to the grid spacing as long as $d / \Delta \geq 1$. . Four levels of resolution have been applied to the agitated flow system: $d / \Delta=1.0,1.6,2.0,2.5$ with all four simulations having the same physical dimensionless parameters given above. The coarsest grid consists of $110^{3}$ cubic cells, the finest of $275^{3}$. Expressed in terms of lattice spacings per impeller diameter, the four resolutions are $D / \Delta=48,76.8,96$, and 120 respectively.

The initial conditions for each simulation are the same: an initial particle configuration is created by letting the 250,000 particles (with $d / \Delta=1.0$ ) settle in a cubic container. Each of the four simulations uses exactly the same dense layer of particles resting on the bottom of the tank with the $(x, y, z)$ locations of the particles scaled according to the specific resolution of the simulation. At moment $t=0$, when fluid and particles have zero velocity, the impeller is set to rotate. The impeller speed is ramped up to its steady 
state value $N$ such that the first impeller revolution takes a time of $2 / N$; beyond $t=2 / N$ the impeller spins at constant speed $N$.

For illustration, we show snapshots of the start-up of the suspension process for the simulation with $d / \Delta=2.0$ in Figure 8 . The velocity magnitude contours in a vertical plane through the center of the tank show liquid being pumped by the impeller in a downward-radial direction. This stream agitates the particles that - as a result - get suspended. Some particles reach the top of the tank within the time needed for eight impeller revolutions. After 16 revolutions particles can be found throughout the entire tank volume, although their distribution is clearly inhomogeneous.

Before discussing the way particles distribute over the tank volume, first the effects of grid resolution on the liquid flow predictions will be discussed. In Figure 9 we show snapshots of the flow close to an impeller blade taken at the same number of impeller revolutions after startup for two different spatial resolutions in terms of liquid and particle velocity vectors. That the overall flow patterns in the two panels of Figure 9 are different is not a direct concern: The impeller generates a mildly turbulent (or transitional) flow so that we expect randomness in the temporal variability of the flow. The left, more resolved panel, however, shows much more fine, small scale detail that seems to be too small to be captured on the coarser grid in the right panel, for example the vortex underneath the hub. We thus anticipate the latter simulation (with $D=48 \Delta$ ) to be under-resolved.

We realize (1) that these are only qualitative observations, and (2) that it might very well be that the simulation in the left panel is under-resolved as well. In fact, in order to fully resolve boundary layers on impeller blades at the current Reynolds number, linear mesh spacings might need to be smaller by an order of magnitude. The boundary layers are, however, not critical for the bulk flow in the tank [33]; the bulk flow is where the main solid-liquid interactions take place.

As a more objective, albeit global, measure for grid convergence the torque $M$ required to spin the impeller is compared between the various grids. If we define the dimensionless torque as $\mathrm{Po}=2 \pi M /\left(\rho N^{2} D^{5}\right)$ it is equivalent to the power number (since power $P=2 \pi N M$ ). Time series of Po 
are shown in Figure 10. They show some time variability as well as a strong effect of the spatial resolution of the simulations. For all cases, a quasi steady state is reached after approximately 20 impeller revolutions. The figure indicates that grid convergence is reached if in the simulation the resolution is such that $D / \Delta \geq 96$ (equivalent to $d / \Delta \geq 2.0$ ). This is an important result. It teaches that the required resolution is in the first place dictated by the liquid flow dynamics. If particles are involved in the flow, we thus need the freedom to choose their size independent of the grid spacing. The results on sedimenting systems in the previous section suggest that with the current mapping procedure particle size independence can be achieved. In the remainder of this paper we will test particle size independence for the more complicated situation (as compared to simple, hindered settling) of a mildly turbulent agitated flow.

Time series of two global particle characteristics are compared for the four resolutions in Figure 11. The left panel shows the number of particles with coordination number of at least $4\left(N_{4}\right)$ over the total number of particles $\left(N_{t}\right)$. The coordination number of a particle is the number of other particles it is in contact with, where contact is defined as overlap $\delta>0$. Since we start from a dense granular bed on the bottom of the tank, the ratio $N_{4} / N_{t}$ is close to one at time zero, and in the subsequent suspension process the ratio $N_{4} / N_{t}$ gets reduced. The fact that it gets reduced to close to zero implies that the solids suspension process under the conditions considered approaches complete suspension over the 60 impeller revolutions of the time series. The second global particle characteristic is the average vertical $(z)$ particle location, plotted as a function of time in the right panel of Figure 11. Both time series show a significant difference between on one side the simulation with $d / \Delta=1$, and on the other side the other three simulations. This is consistent with the results for the power number (Figure 10) where also the $d / \Delta=1$ stood out from the others. The difference as observed in power number for $d / \Delta=1.6$ with the finer simulations, however, does not reflect in markedly different global particle behavior. 
In Figures 12 and 13 a more local characterization of the suspension process is presented. Here we look into what happens around time $t N=20$. Considering this moment in time when the suspension process is still in progress (see Figure 11) is a more interesting and critical test for assessing resolution effects than looking at the fully suspended, quasi steady state. Figure 12 shows instantaneous realizations at $t N=20$ of the situation in a vertical cross section through the center of the tank for the four simulations considered. The way individual particles intersect that plane is indicated by the white disks; the colors indicate the instantaneous Eulerian solids volume fraction fields as obtained through mapping from the particle locations and the figure thus serves as an illustration of how the mapping operation works. The particles concentrate under the impeller since the flow there is relatively weak (see Figure 9). There are more particles close to the bottom for the least resolved simulation $(d / \Delta=1)$ compared to the other simulations, in line with the results in Figure 12. No systematic differences between the other simulations can be concluded from the snapshots in Figure 12. For this reason average solids volume fraction fields over the period $15 \leq t N \leq 20$ are shown in Figure 13. They confirm the least level of solids suspension for $d / \Delta=1$ and tentatively - not significantly - show less particles in the bottom region and in the solids cone underneath the impeller the higher the spatial resolution of a simulation. We conclude from these results that a decent level of grid independence for this two-phase system under its current conditions is achieved for $d / \Delta \geq 2$.

It should be realized that the primary purpose of this paper is to establish a procedure for solidliquid simulations in which the resolution can be tailored independently to the needs of fluid as well as solids mechanics. The level of realism of the simulation results depends on much more than on (the elimination of) grid effects only. One of the choices we made was for the Wen \& Yu drag force correlation [24]; another choice was the incorporation of lubrication forces. To judge the impact of these specific choices, two additional simulations (with resolution $d / \Delta=1.6$ ) were performed. One using the Van der Hoef et al drag force expression [25] instead of the Wen \& Yu correlation, the other without 
lubrication forces. Results are in Figure 14. They have been presented in the same way as the results in Figures 12 and 13 for the base-cases.

The effects of the lubrication force are very significant: suspending the solids is much easier without activated lubrication forces. Mobilizing the granular bed requires pulling apart connected particles. In this process the lubrication force is an attractive force. The change in drag correlation is less drastic but still visible: suspension of the solids after 20 impeller revolutions has advanced less with the Van der Hoef et al correlation as compared to the Wen \& Yu correlation.

\section{Conclusions and outlook}

In this paper we have assessed a procedure - based on the lattice-Boltzmann method - for performing Eulerian-Lagrangian simulations of dense solid-liquid systems with unresolved particles. We focused on the effects of spatial resolution. Two flow systems were considered: (1) settling of particles under gravity towards a solid, horizontal wall; (2) an agitated tank with particles getting suspended by a transitional (Reynolds number 4,000) liquid flow. The modelling approach is relatively simple: drag and lubrication are the only hydrodynamic forces considered. The drag force depends on the local solids volume fraction through the Wen \& Yu correlation [24] which we justify because we are dealing with solid-liquid systems (that have modest Stokes numbers). Particle collisions are smooth. The solids and liquid dynamics are two-way coupled except for particle rotation which is one-way (only fluid to solid) coupled.

The main conclusion of the sedimentation simulations is that the results in terms of average settling speed as well as particle velocity fluctuations are independent of the particle size relative to the lattice spacing if we use mapping functions with a fixed width relative to the particle size. The dependency of the hindered settling speed as a function of the average solids volume fraction is in reasonable agreement with empirical correlations from the literature [31].

The independence of the particle size relative to the grid spacing is an important feature if the grid resolution is decided by factors other than the solids dynamics. In the case of the mixing tank, the 
transitional flow generated by the impeller is decisive for the choice of resolution and grid effects first and foremost show up for the torque required to spin the impeller. Global and local parameters characterizing the solids suspension process showed grid-independent behavior beyond a certain spatial resolution. It should be realized that simulation results - including those that approach grid-independence - depend on the choice of the width of the mapping function relative to the particle size. Further study is needed to explore how to objectively make this choice and to what extent this choice is regime (solids volume fraction, Reynolds number, Stokes number) dependent.

Given the relative simplicity of the way solids dynamics has been modelled and coupled to the liquid dynamics there is ample room for model refinement. An important question in this respect is, however, how to judge if model refinement leads to improvement of the level of realism of the simulations. In our opinion we need detailed experiments for this, e.g. based on visualization and optical velocity measurements in refractive index matched solid-liquid systems [19]. For example, an experiment along these lines in a mixing tank would be able to decide if the role of the lubrication force is indeed as important as shown by the numerical results in this paper, or if there are advantages of using one formulation of a drag force correlation over another. 


\section{References}

[1] Stokes GG. Mathematical and Physical Papers, Volumes I-V. Cambridge: Cambridge University Press; 1901.

[2] Richardson JF, Zaki WN. Sedimentation and fluidisation. Part 1. Trans. Inst. Chem. Engrs. 1954; 32 : $35-53$.

[3] Zwietering ThN. Suspending of solid particles in liquid by agitators. Chem. Engng. Sc. 1958; 9, 244 253.

[4] Uhlmann M. Interface-resolved direct numerical simulation of vertical particulate channel flow in the turbulent regime. Phys. Fluids 2008; 20: 053305.

[5] Lucci F, Ferrante A, Elghobashi S. Modulation of isotropic turbulence by particles of Taylor lengthscale size. J. Fluid Mech. 2010; 650: 5-55.

[6] Derksen JJ. Highly resolved simulations of solids suspension in a small mixing tank. AIChE J. 2012; 58: $3266-3278$

[7] Wachs A, Hammouti A, Vinay G, Rahmani M. Accuracy of finite volume/staggered grid distributed Lagrange multiplier/fictitious domain simulations of particulate flows. Comp. Fluids 2015; 115: $154-172$.

[8] Kidanemariam, A.G., Uhlmann, M. Formation of sediment patterns in channel flow: minimal unstable systems and their temporal evolution. J. Fluid Mech. 2017; 818: 716-743.

[9] Beetstra R, Van der Hoef MA, Kuipers JAM. Drag force of intermediate Reynolds number flow past mono- and bi-disperse arrays of spheres. AIChE J. 2007; 52: 489-501.

[10] Tenneti S, Garg R, Subramaniam S. Drag law for monodisperse gas-solid systems using particleresolved direct numerical simulation of flow past fixed assemblies of spheres. Int. J. Multiphase Flow 2011; 37: 1072-1092.

[11] Rubinstein GJ, Derksen JJ, Sundaresan S. Lattice-Boltzmann simulations of low-Reynolds number flow past fluidized spheres: effect of Stokes number on drag force. J. Fluid Mech. 2016; 788: 576601.

[12] Wylie JJ, Koch DL, Ladd AJC. Rheology of suspensions with high particle inertia and moderate fluid inertia. J. Fluid Mech. 2003; 480: 95-118.

[13] Clift R, Grace JR, Weber ME. Bubbles, Drops, and Particles. New York: Academic Press; 1978.

[14] Maxey MR, Riley JJ. Equation of motion for a small rigid sphere in a nonuniform flow. Phys. Fluids 1983; 26: 883-889.

[15] Deen NG, Van Sint Annaland M, Van der Hoef MA, Kuipers JAM. Review of discrete particle modeling of fluidized beds. Chem. Eng. Sc. 2007; 62: 28-44. 
[16] Derksen JJ. Numerical simulation of solids suspension in a stirred tank. AIChE J. 2003; 49: 27002714.

[17] Capecelatro J, Desjardins O. An Euler-Lagrange strategy for simulating particle-laden flow., J. Comp. Phys. 2013; 238: 1-31.

[18] Derksen JJ. Assessing Eulerian-Lagrangian simulations of dense solid-liquid suspensions settling under gravity. Comp. \& Fluids 2016 in press; http://dx.doi.org/10.1016/j.compfluid.2016.12.017.

[19] Genghong Li, Zhengming Gao, Zhipeng Li, Jiawei Wang, Derksen JJ. Particle-resolved PIV experiments of solid-liquid mixing in a turbulent stirred tank. AIChE J. 2017; under review.

[20] Sungkorn R, Derksen JJ. Simulations of dilute sedimenting suspensions at finite-particle Reynolds numbers. Phys. Fluids 2012; 24: 123303.

[21] Schiller L, Naumann A. Uber die grundlagenden Berechnungen bei der Schwerkraftaufbereitung. Ver. Deut. Ing. Z. 1933; 77: 318-320.

[22] Sankaranarayanan K, Sundaresan S. Lattice Boltzmann simulation of two-fluid model equations. Ind. Eng. Chem. Res. 2008; 47: 9165-9173.

[23] Gidaspow D. Multiphase Flow and Fluidization. San Diego: Academic Press: 1994.

[24] Wen CY, Yu YH. Mechanics of fluidization. Chem. Engng. Prog. 1966; 62; 100-111.

[25] Van der Hoef MA, Beetstra R, Kuipers JAM. Lattice-Boltzmann simulations of low-Reynoldsnumber flow past mono- and bidisperse arrays of spheres: results for the permeability and drag force. J. Fluid Mech. 2005; 528, 233-254.

[26] Derksen JJ, Sundaresan S. Direct numerical simulations of dense suspensions: wave instabilities in liquid-fluidized beds. J. Fluid Mech. 2007; 587: 303-336.

[27]Deen NG, Van Sint Annaland M, Kuipers JAM. Multi-scale modeling of dispersed gas-liquid twophase flow. Chem. Eng. Sc. 2004; 59: 1853-1861.

[28] Kim S, Karrila SJ. Microhydrodynamics: Principles and selected applications. Boston: ButterworthHeinemann; 1991.

[29]Deen WM. Analysis of transport phenomena. New York: Oxford University Press; 1998.

[30] Shardt O, Derksen JJ. Direct simulations of dense suspensions of non-spherical particles. Int. J. Multiphase Flow 2012; 47: 25-36.

[31]Di Felice R. The voidage function for fluid-particle interaction systems. Int. J. Multiphase Flow. 1994; 20: 153-159.

[32] Guazzelli É, Hinch J. Fluctuations and instability in sedimentation. Annu. Rev. Fluid Mech. 2011; 43: 97-116.

[33]Derksen J, Van den Akker HEA. Large-eddy simulations on the flow driven by a Rushton turbine. AIChE J. 1999; 45: 209-221. 
Figures

Figure 1. Mixing tank geometry: top view and side view. The origin of the Cartesian coordinate is in the center of the bottom wall.
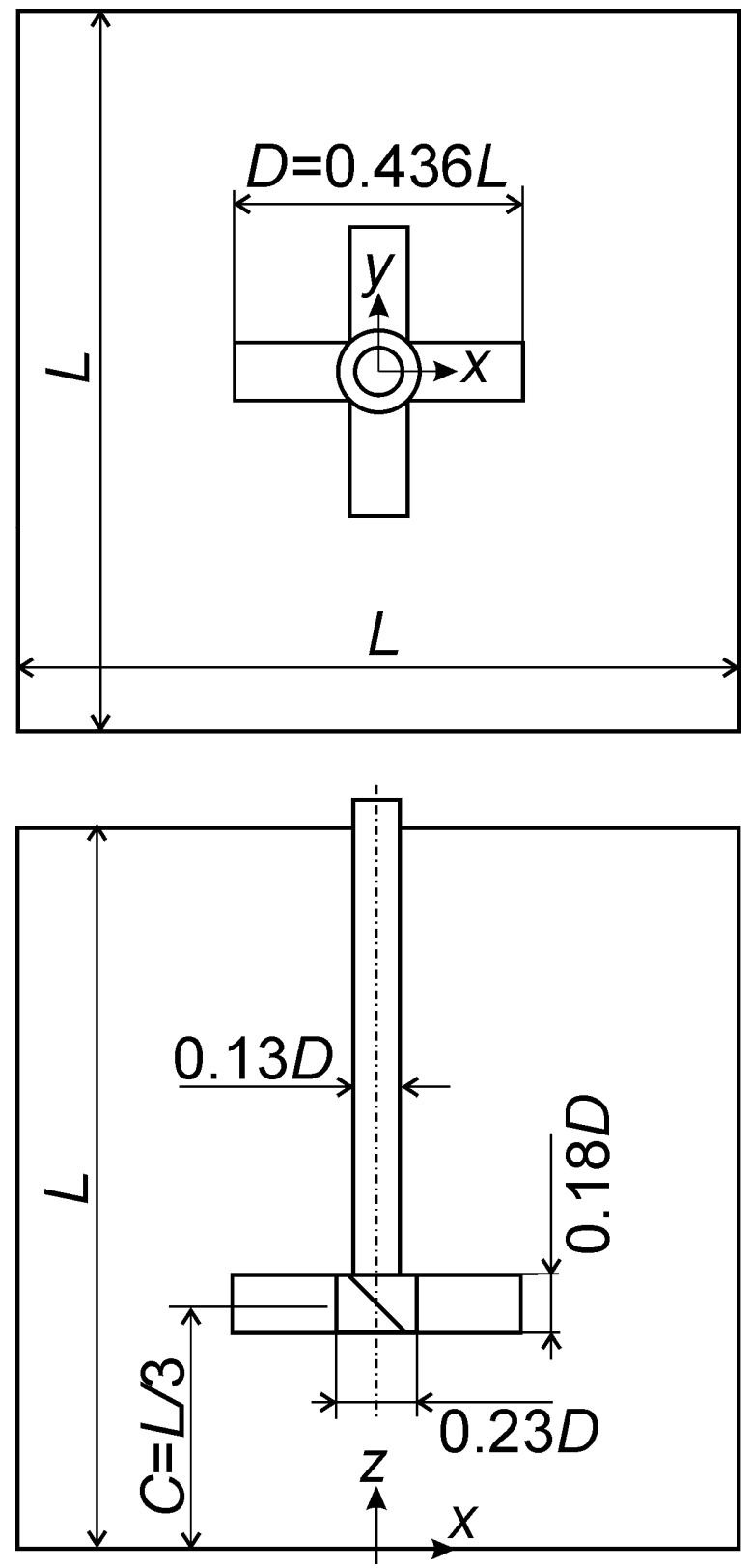
Figure 2. Base-case for hindered settling at two resolutions: left $d=1.1 \Delta$, right $d=2.2 \Delta$. Instantaneous realizations $t u_{\infty} / d=13.4$ after start-up. Cross sections through the middle of the flow domain. Black vectors: interstitial liquid velocity; red vectors: velocity of particles in a $4 d$ thick layer in the middle of the domain. The reference vector indicates the single-particle settling velocity $u_{\infty}$.

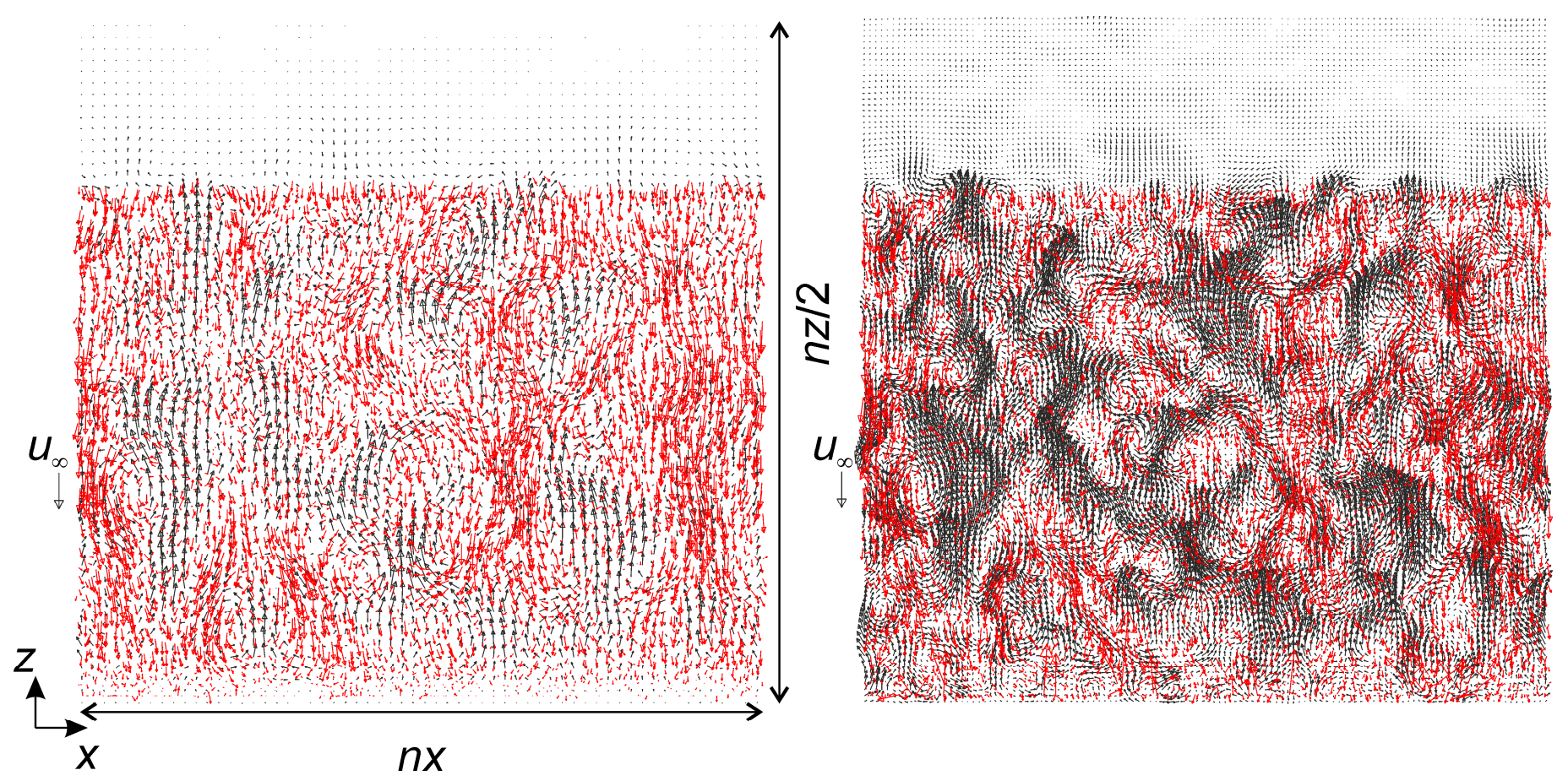


Figure 3. Left: the solid blue curve is the vertical pressure profile averaged over the lateral directions $\langle p\rangle$; the dashed line is to show slope -0.25 . Right: solids volume fraction distribution in a vertical cross section. Instantaneous realization at $t u_{\infty} / d=35.9$. Base-case with $d=1.1 \Delta$.

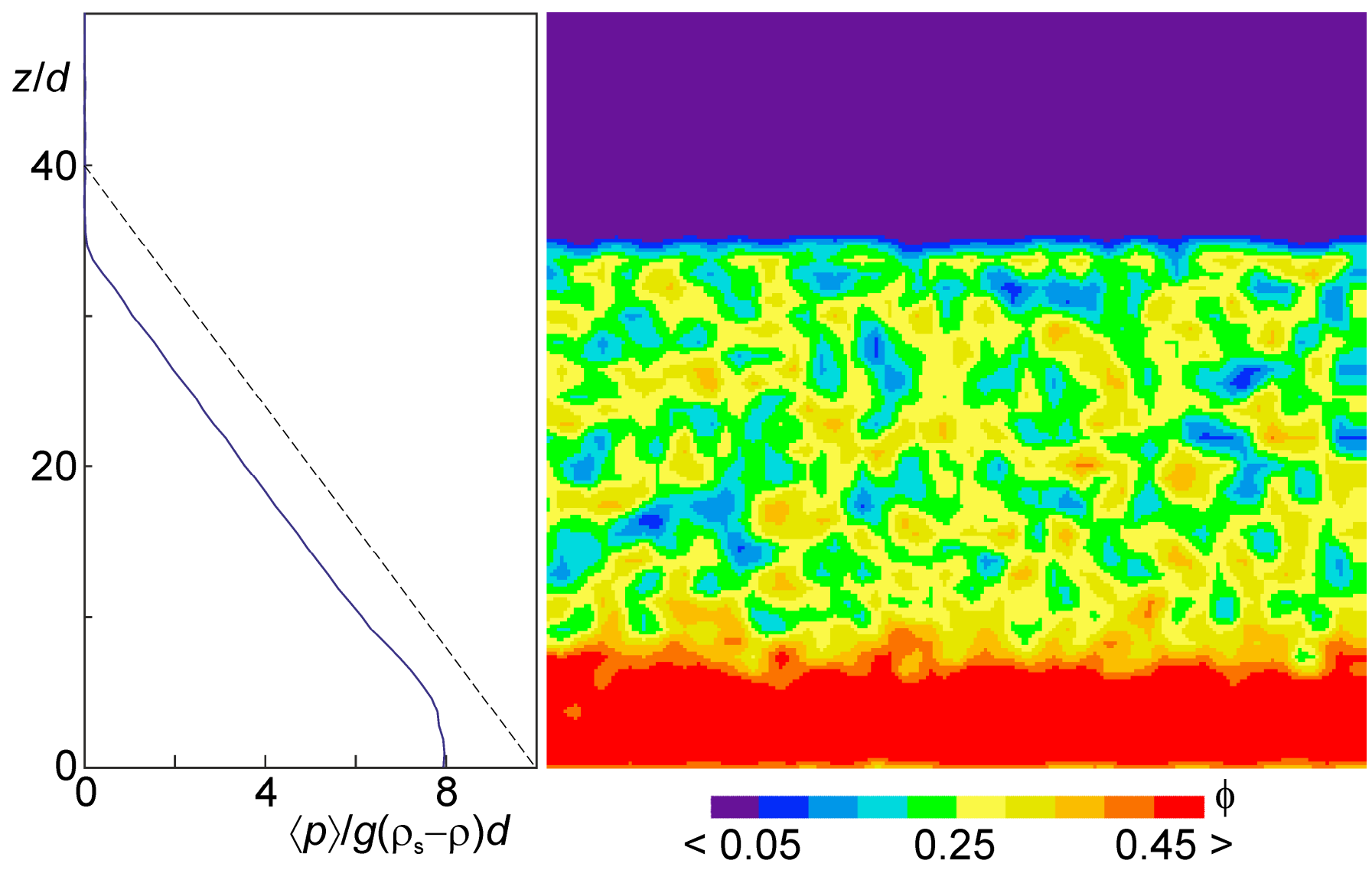


Figure 4. Time series of the vertical interface location according to two methods as explained in the text. Base-case with $d=1.1 \Delta$.

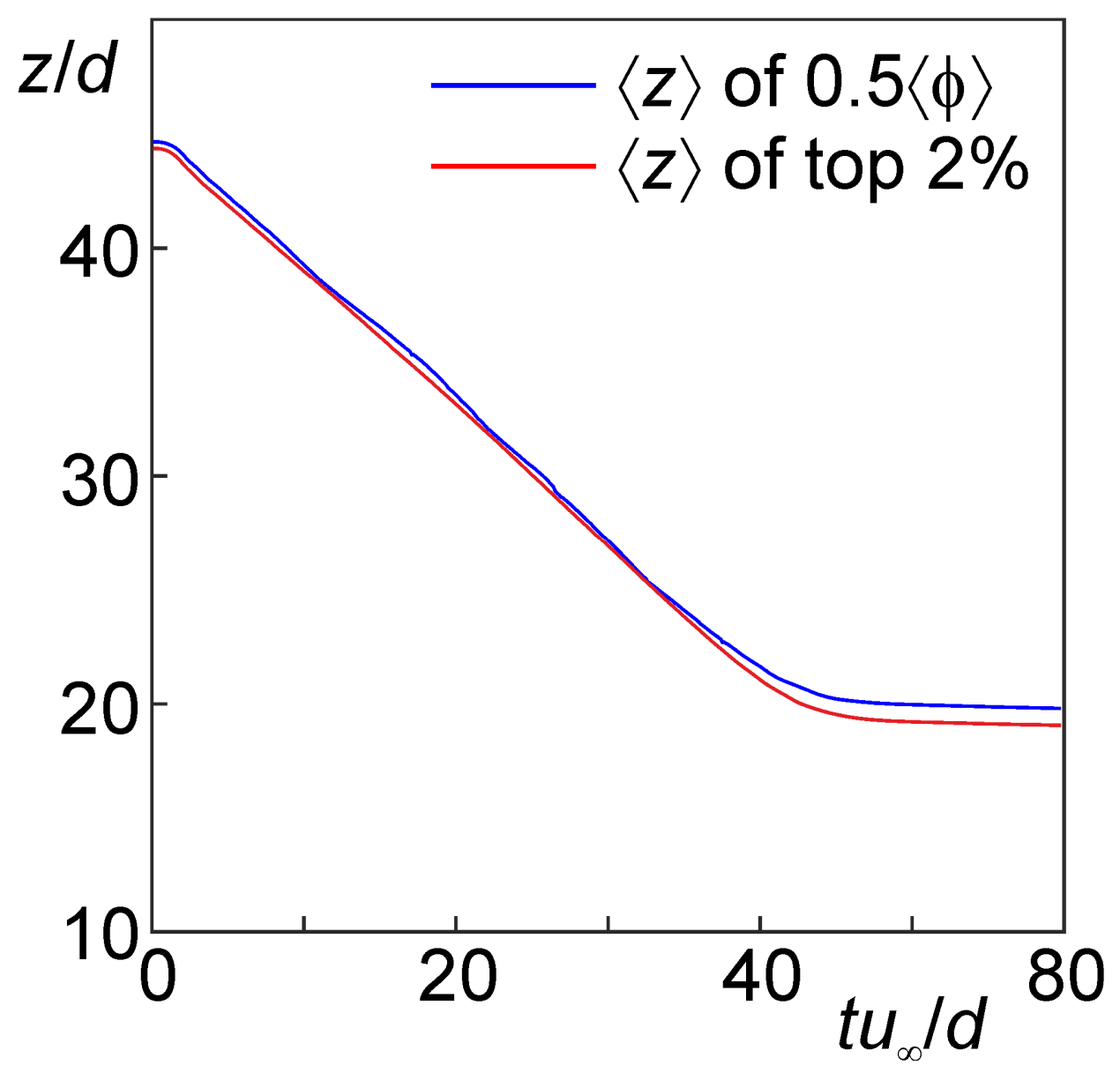


Figure 5. Left: hindered settling velocity as a function of solids volume fraction for $\operatorname{Re}_{\infty}=2.89$, $\rho_{s} / \rho=2.50, d / \Delta=1.1$, and $\lambda / d=1.5$. Right: same data as in left figure with $n=\ln \left(\frac{u_{s} / u_{\infty}}{1-\langle\phi\rangle}\right) ;$ the curve is $n=4.7-0.65 \exp \left[-(1.5-x)^{2} / 2\right]$ with $x={ }^{10} \log \left(u_{s} d / v\right)$ due to Di Felice [30].
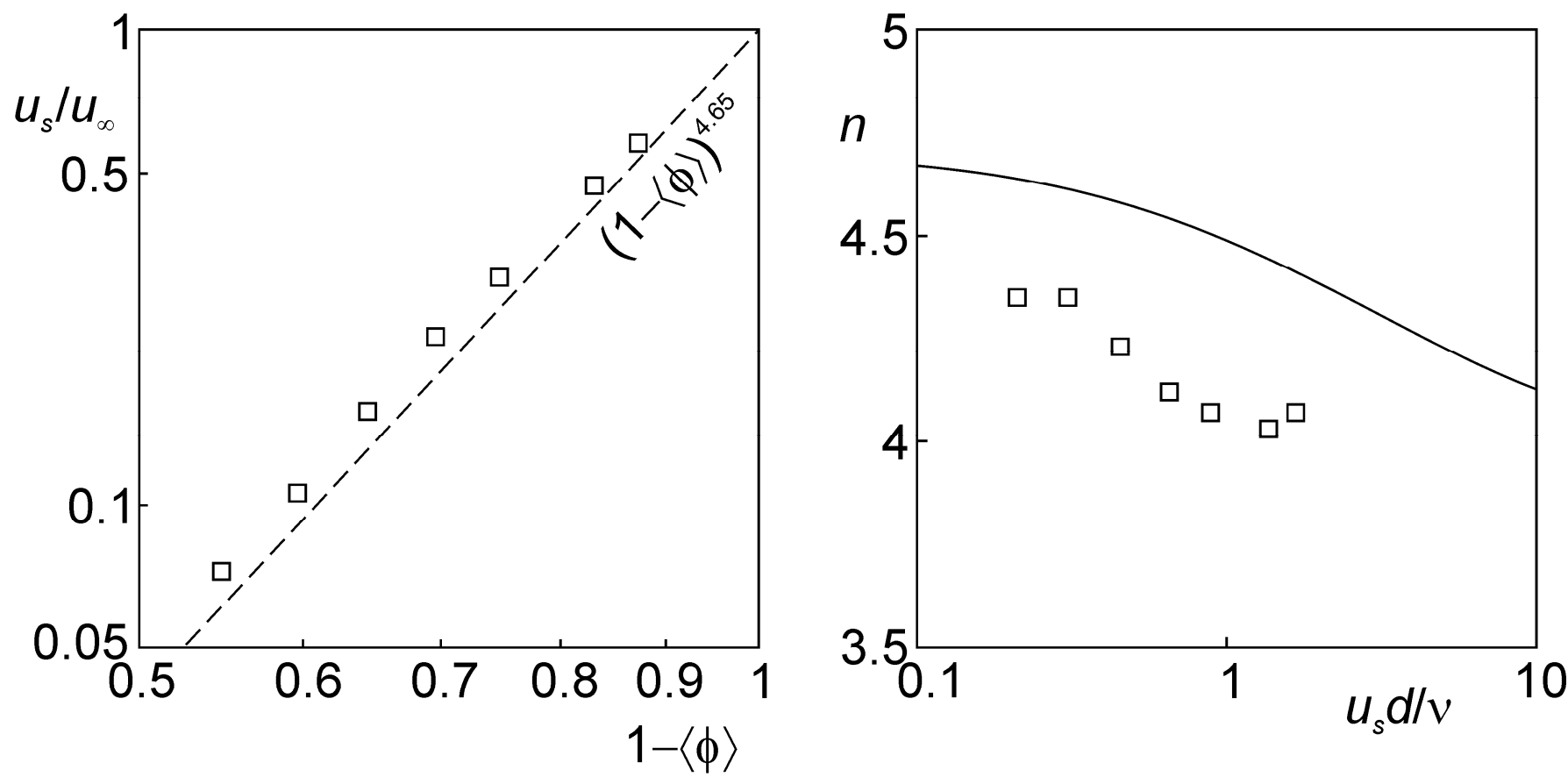
Figure 6. Settling speed as a function of numerical parameters. Left: effect of the half-width of the mapping function $(\lambda)$ for $d=1.1 \Delta$. Right: effect of particle size relative to grid spacing $(d / \Delta)$ for $\lambda=1.5 d$. In all cases, $\operatorname{Re}_{\infty}=2.89, \rho_{s} / \rho=2.50 ;\langle\phi\rangle$ as indicated.
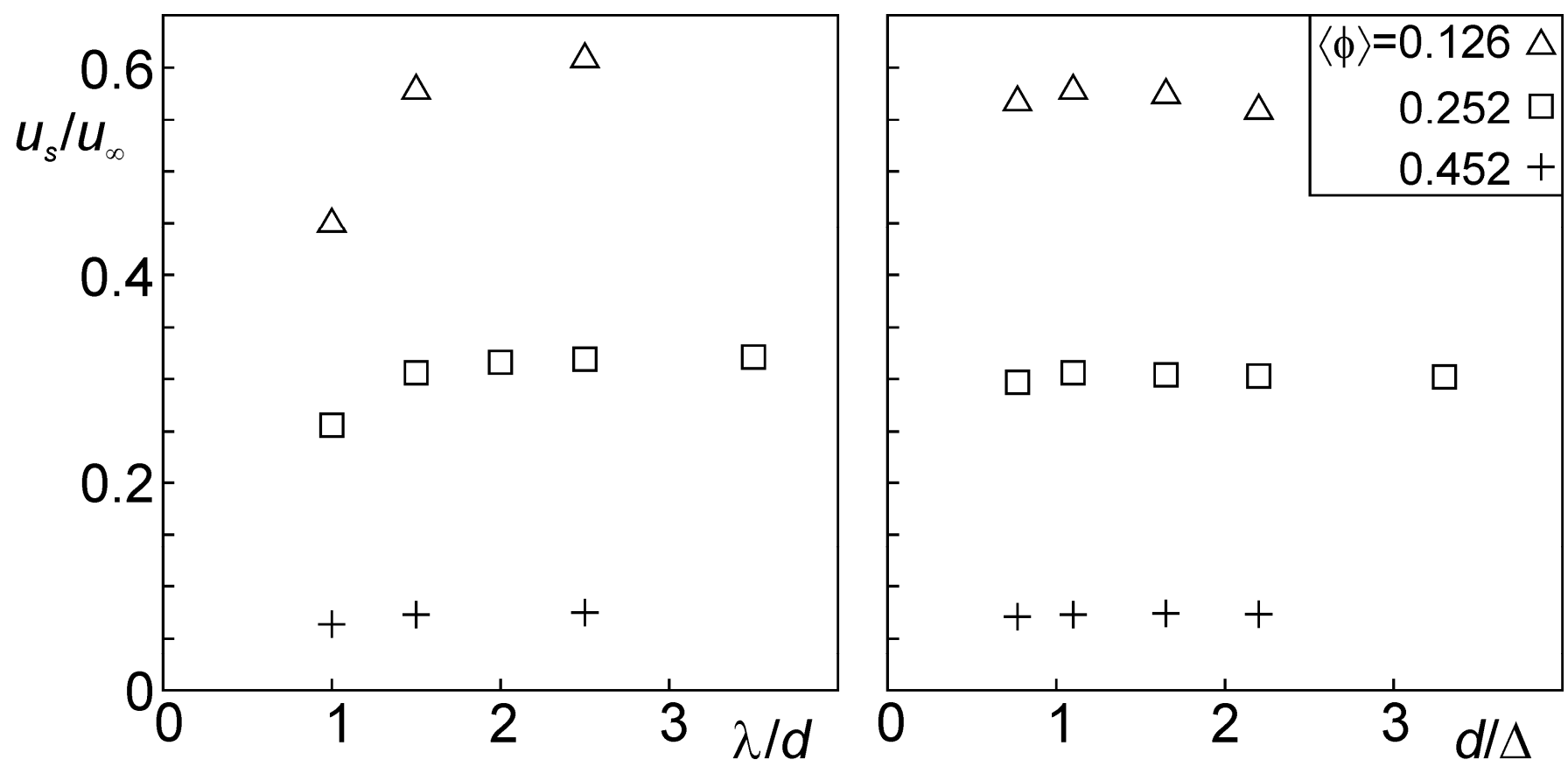
Figure 7. Time series of particle velocities fluctuation levels (root-mean-square values) in horizontal and vertical direction in a horizontal layer with thickness $8 d$ centered at $z=n z / 3$. Left: effect of $\lambda$ for $d=1.1 \Delta$. Right: effect of $d$ with $\lambda=1.5 d$. $\operatorname{Re}_{\infty}=2.89, \rho_{s} / \rho=2.50 ;\langle\phi\rangle=0.252$.

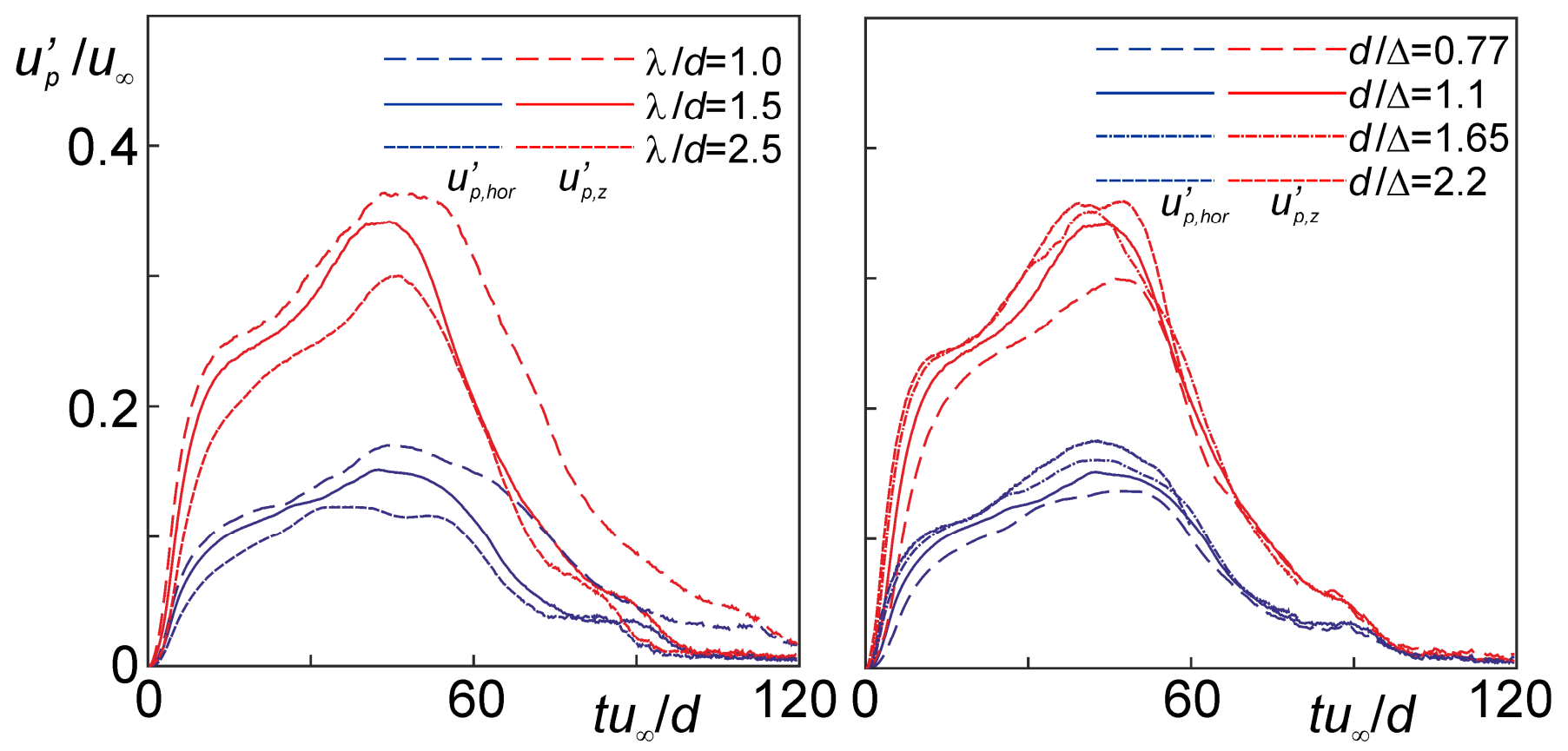


Figure 8. Start of the solids suspension process in the mixing tank in terms of particle positions and velocity magnitude contours. In the two right panels only particles in a slab through the center of width $0.2 T$ are displayed in order to better see inside the system. Base-case conditions with a resolution $d / \Delta=2.0$.
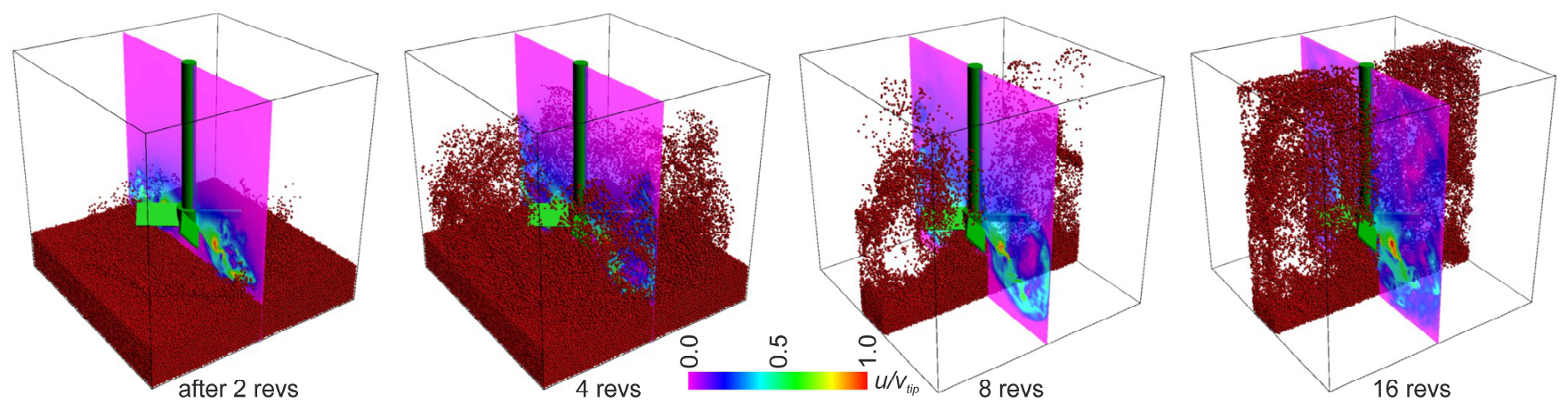
Figure 9. Impressions of instantaneous flow near the impeller (area indicated in grey in the right panel). Black vectors are liquid velocity, red vectors particle velocity. The blue dots are the points used in the immersed boundary method to represent the impeller. The left panel $(d / \Delta=2.0)$ has a twice as high resolution as the middle panel $(d / \Delta=1.0)$. Base-case conditions; 29 impeller revolutions after start-up.
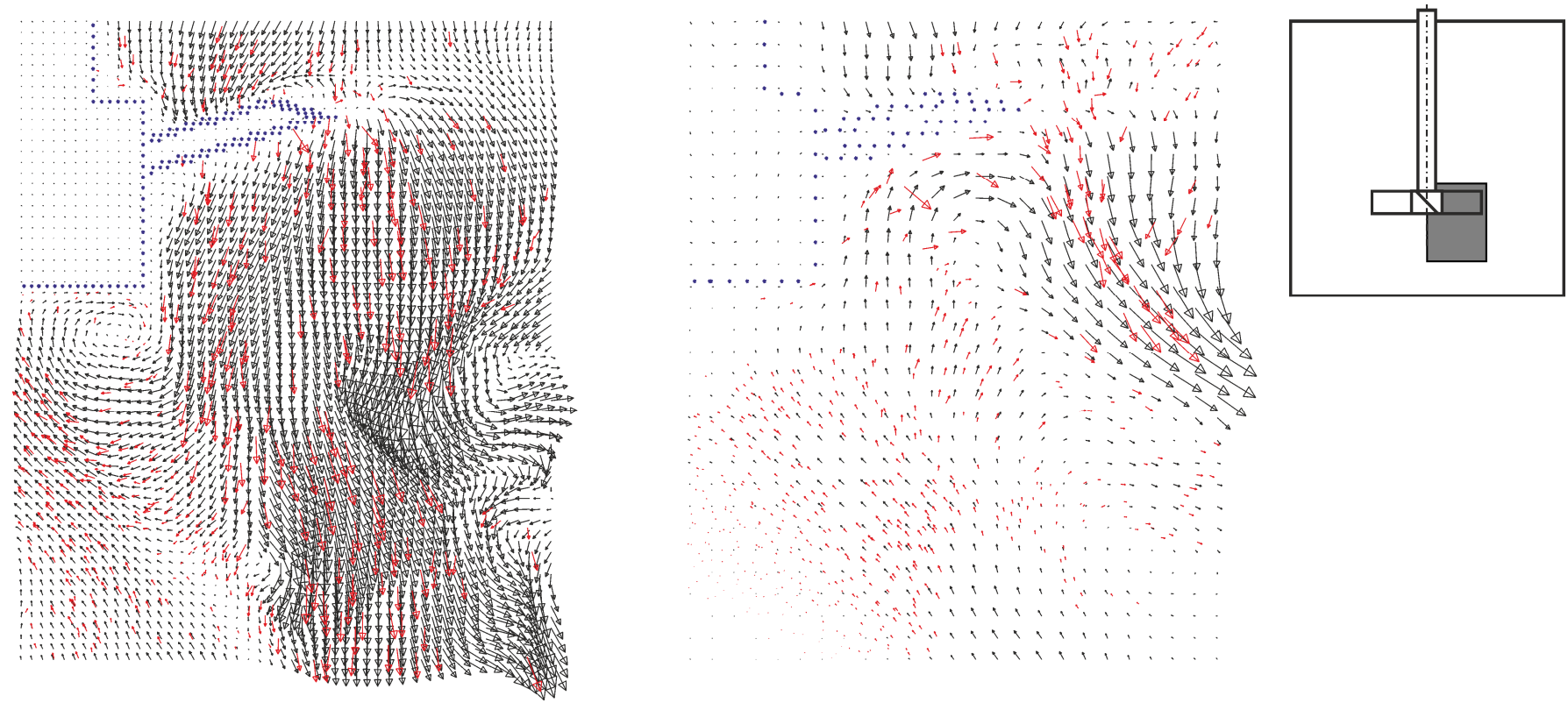
Figure 10. Left: time series of power number Po for simulations with different resolution; right: timeaveraged power number determined for $t N \geq 20$.

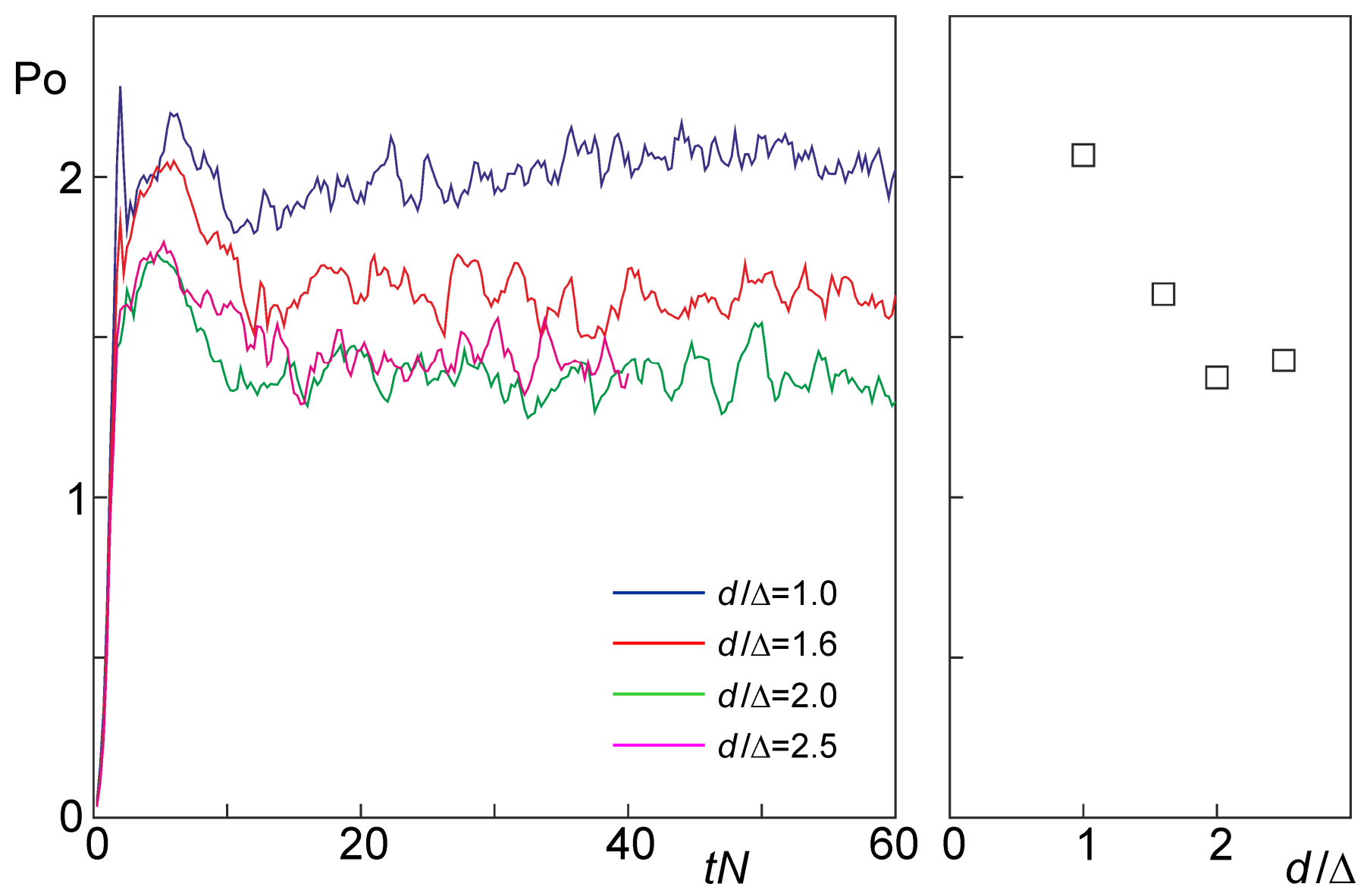


Figure 11. Time series of the fraction of particles having a coordination number of 4 or higher (left) and the average vertical location of the particles (right) for the four different resolutions.
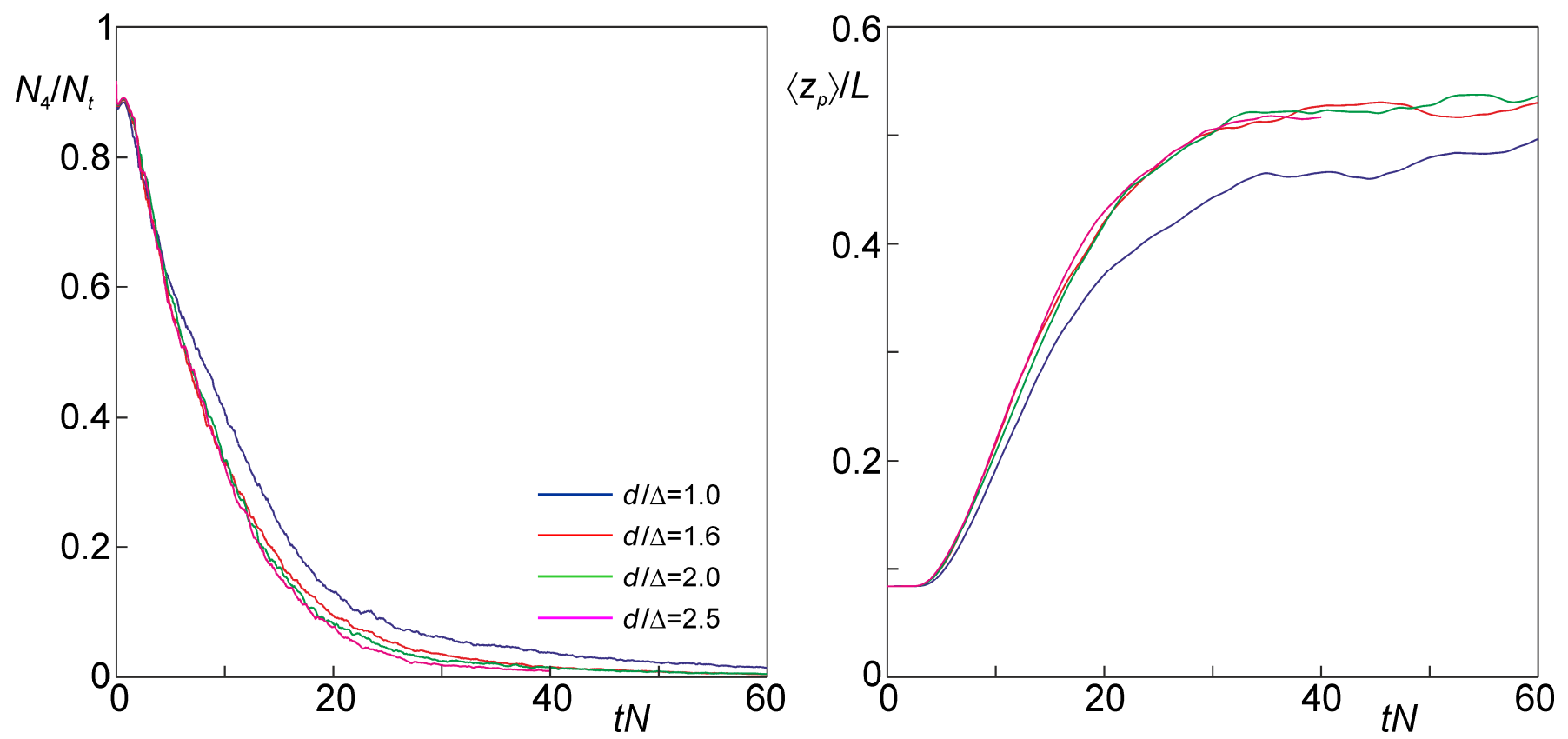
Figure 12. Instantaneous realizations of the solids volume fraction in a vertical plane through the center of the tank at $t N=20$. The white dots are cross sections of individual particles. Increasing resolution from left to right: $d / \Delta=1.0,1.6,2.0$, and 2.5 respectively.
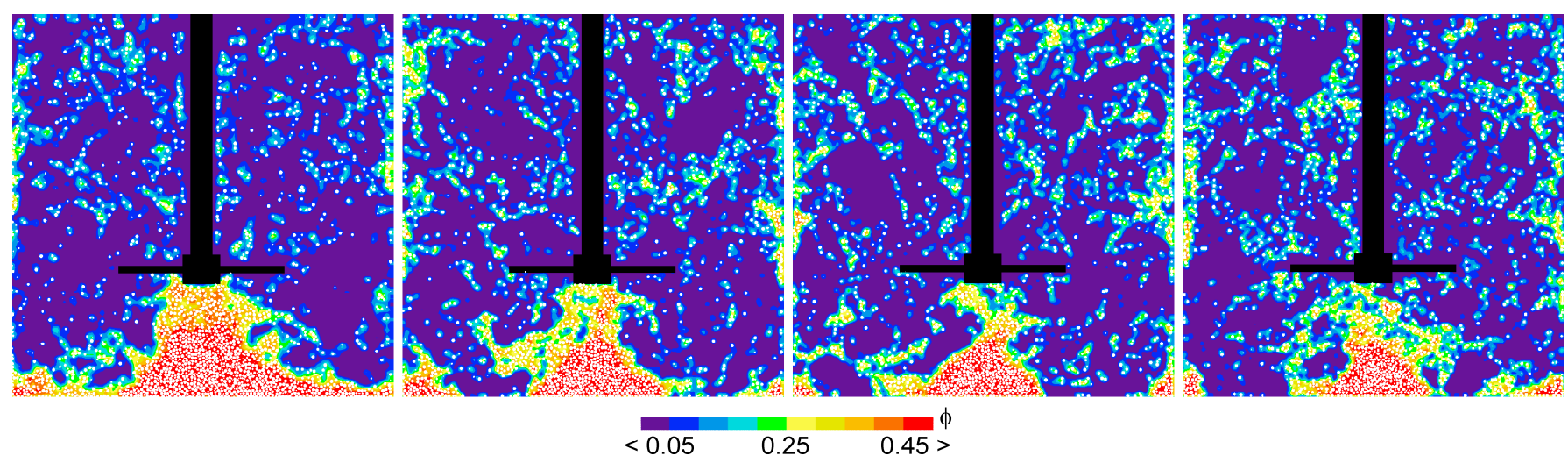
Figure 13. Time-averaged solids volume fraction in a vertical plane through the center of the tank. Averaging over five impeller revolutions $15 \leq t N \leq 20$. Increasing resolution from left to right: $d / \Delta=1.0$, $1.6,2.0$, and 2.5 respectively.
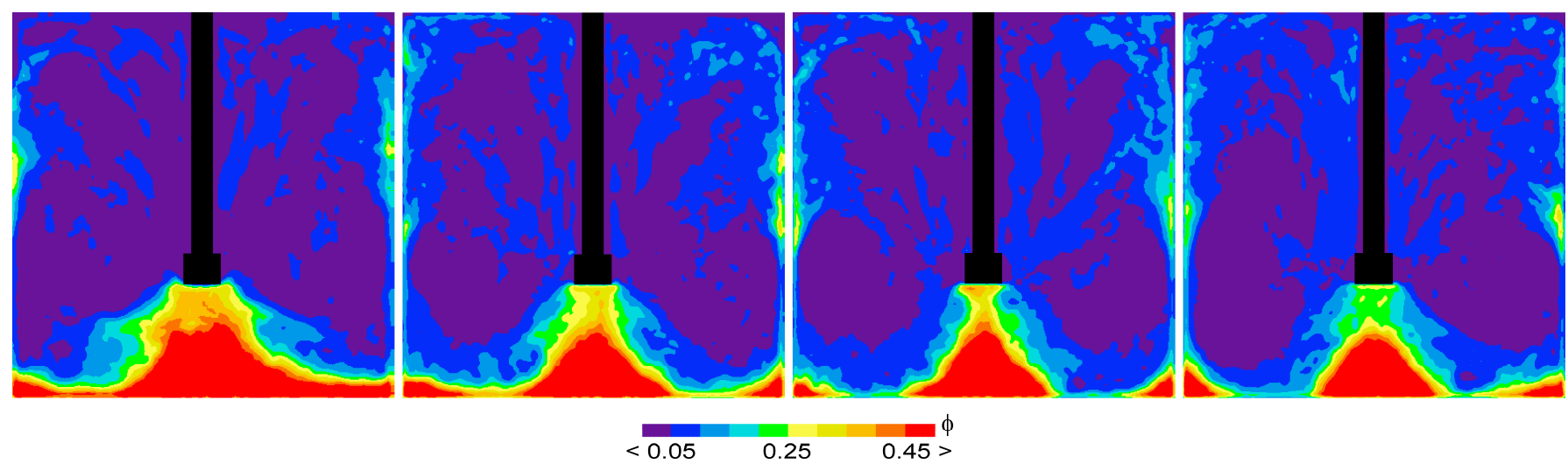
Figure 14. Top row: instantaneous realizations of the solids volume fraction in a vertical plane through the center of the tank at $t N=20$; bottom row: time-averaged $(15 \leq t N \leq 20)$ solids volume fraction. Left: Van der Hoef et al [24] instead of Wen \& Yu drag correlation; right: lubrication force switched off. In both cases $d / \Delta=1.6$
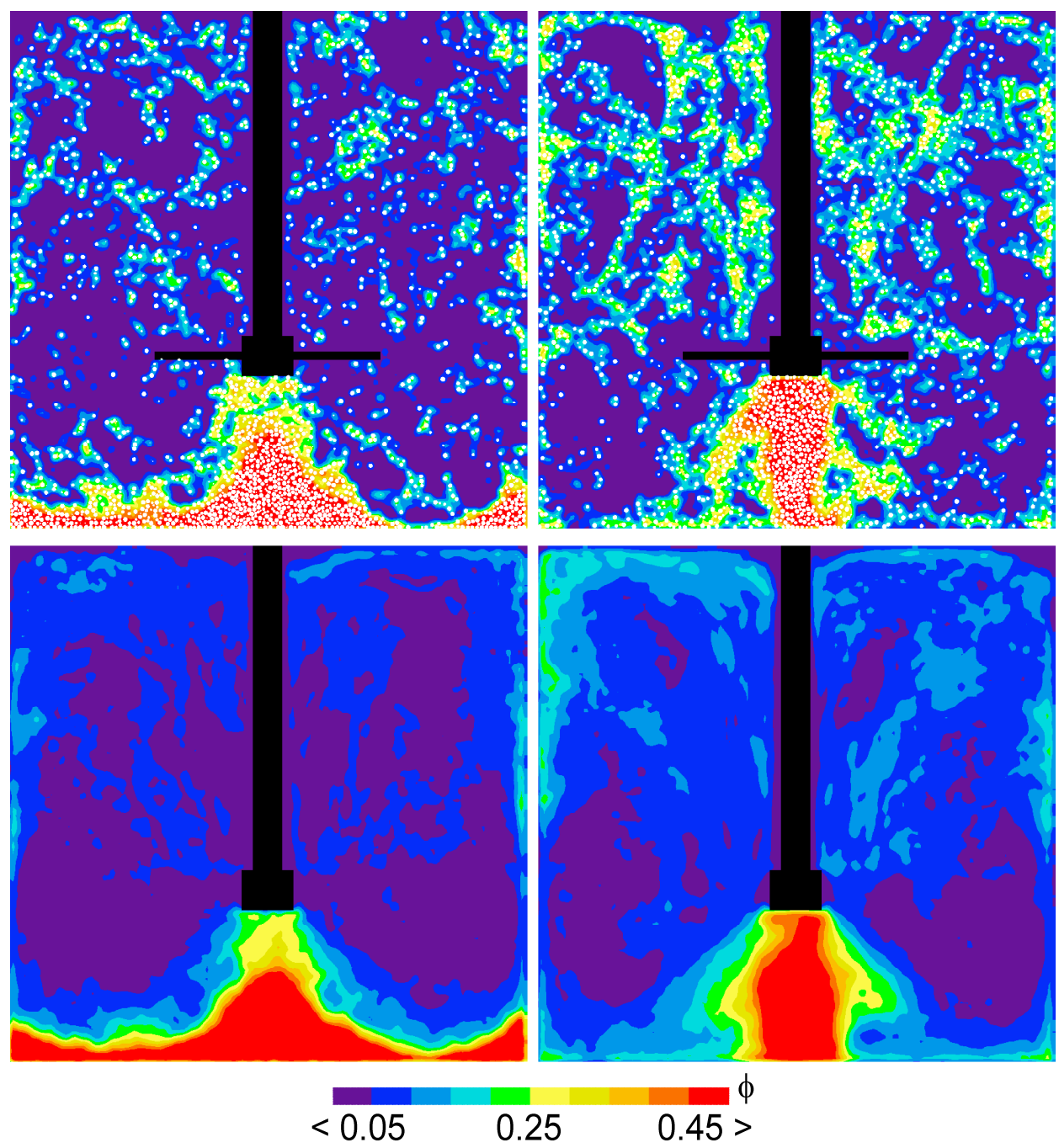